\title{
openheart Prevalence and prognostic implication of iron deficiency and anaemia in patients with severe aortic stenosis
}

Anette Borger Kvaslerud, ${ }^{1,2}$ Amjad lqbal Hussain, ${ }^{1,3}$ Andreas Auensen, ${ }^{1,2}$ Thor Ueland, ${ }^{4,5}$ Annika E Michelsen, ${ }^{1,4}$ Kjell Ingar Pettersen, ${ }^{3}$ Pål Aukrust, ${ }^{1,6}$ Lars Mørkrid, ${ }^{1,7}$ Lars Gullestad, ${ }^{1,2,3}$ Kaspar Broch ${ }^{2,3}$

\begin{abstract}
- Additional material is published online only. To view please visit the journal online (http://dx.doi.org/10.1136/ openhrt-2018-000901).
\end{abstract}

To cite: Kvaslerud $A B$, Hussain Al, Auensen A, et al. Prevalence and prognostic implication of iron deficiency and anaemia in patients with severe aortic stenosis. Open Heart 2018;5:e000901.

doi:10.1136/

openhrt-2018-000901

Preliminary results from this study have been presented as a poster presentation at the ESC Heart Failure Congress in Vienna in May 2018 prior to submission.

Received 9 July 2018

Revised 3 October 2018

Accepted 12 November 2018

(C) Author(s) (or their employer(s)) 2018. Re-use permitted under CC BY-NC. No commercial re-use. See rights and permissions. Published by BMJ.

For numbered affiliations see end of article.

Correspondence to Dr Anette Borger Kvaslerud; a.b. kvaslerud@medisin.uio.no

\section{ABSTRACT}

Objective The aim of this study was to evaluate the prevalence and prognostic implication of iron deficiency (ID) and anaemia in patients with severe aortic stenosis (AS).

Methods In an observational study of consecutive patients referred for aortic valve replacement (AVR), we assessed a wide range of biomarkers of iron status, including the definition of ID commonly applied in patients with chronic heart failure (ferritin $<100 \mu \mathrm{g} / \mathrm{L}$ or ferritin $100-299 \mu \mathrm{g} / \mathrm{L}$ with a transferrin saturation $<20 \%$ ). The endpoints were short-term (one-year) and long-term (median 4.7 years, IQR: 3.8-5.5) mortality and major adverse cardiovascular events (MACE) within the first year after inclusion.

Results 464 patients were included in this substudy. 91 patients $(20 \%)$ received conservative treatment and 373 patients $(80 \%)$ received AVR. ID was detected in 246 patients (53\%). 94 patients (20\%) had anaemia. Patients with ID had an overall worse clinical profile than patients without ID. During follow-up, 129 patients (28\%) died. Neither ID as defined above, soluble transferrin receptor nor hepcidin were associated with short-term or long-term mortality or MACE independent on treatment allocation. Anaemia was associated with one-year mortality in conservatively treated patients.

Conclusions ID and anaemia are prevalent in patients with severe AS. In our cohort, ID did not provide independent prognostic information on top of conventional risk factors. More studies are required to determine how to correctly diagnose ID in patients with AS.

Trial registration number NCT01794832.

\section{INTRODUCTION}

Iron deficiency (ID) is the most prevalent nutritional disorder in the world ${ }^{1}$ and a common cause of anaemia. ${ }^{2}$ Although ID traditionally is linked to anaemia, ID is prevalent even in the absence of anaemia and in itself limits function and survival. ${ }^{34}$ ID is a frequent feature of various chronic diseases, and up to $50 \%$ of patients with heart failure (HF) have ID. ${ }^{56}$ In HF, ID has been associated with reduced quality of life (QoL) and exercise tolerance and constitutes an independent

\section{Key questions}

What is already known about this subject?

- Iron deficiency is detrimental in patients with chronic heart failure and possibly also in patients with other cardiovascular diseases such as coronary artery disease. Anaemia has been associated with adverse outcomes in patients with aortic stenosis, but no previous study has investigated the prevalence and prognostic implications of iron deficiency in patients with aortic stenosis regardless of haemoglobin level.

What does this study add?

- This study provides new insight on the significance of iron deficiency in patients with severe aortic stenosis. Our results show that iron deficiency and anaemia are common comorbidities in patients with severe aortic stenosis, with a prevalence of $53 \%$ and $20 \%$, respectively. Iron deficient patients have worse clinical profile, but iron deficiency was not associated with increased mortality or morbidity. Anaemia was associated with one-year mortality in conservatively treated patients.

How might this impact on clinical practice?

- Knowledge of the significance of comorbidities such as anaemia and iron deficiency is valuable for clinicians treating patients with aortic stenosis. Although more studies are indicated to confirm our findings, there is currently no compelling basis for recommending treatment of isolated iron deficiency in patients with severe aortic stenosis.

predictor of morbidity, mortality and cardiac transplantation. ${ }^{5-8}$ Conversely, randomised controlled trials have shown that correction of ID with intravenous iron therapy improves functional capacity, QoL and six-minute walk distance. ${ }^{910}$ Newer data also suggest that ID is detrimental in patients with coronary artery disease (CAD), ${ }^{11} 12$ pulmonary hypertension $^{13} 14$ and possibly in patients undergoing cardiac surgery. ${ }^{15}$

The diagnostics of ID in patients with chronic disorders, and in particular those characterised by low-grade systemic 
inflammation, may be challenging. The most commonly used definition of ID in cardiology (ID; ferritin $<100 \mathrm{ng}$ / $\mathrm{mL}$ or ferritin $100-300 \mathrm{ng} / \mathrm{mL}$ and transferrin saturation $<20 \%$ ) derives from large-scale studies of $\mathrm{HF}$ and includes both absolute and functional ID. ${ }^{16}$ This definition is based on the assumption that, due to chronic disease, the ferritin cut-point for ID should be higher in patients with HF than in the general population. The validity of this definition has not until recently been evaluated, ${ }^{17}$ and although the same argument for choosing a higher ferritin cut-point could be made in other chronic cardiovascular diseases such as aortic stenosis (AS), the transferability of this definition is unclear.

Patients with AS typically have numerous comorbidities, including anaemia, which occurs in 22\%-57\% of patients. ${ }^{18-21}$ Anaemia in patients with AS is associated with reduced QoL, poorer functional capacity and reduced survival. ${ }^{18}{ }^{19}$ However, in patients with AS, we do not know the prevalence of non-anaemic ID or the consequences of having ID.

In this study we examine the prevalence and prognostic implication of ID and anaemia in patients with severe AS (regardless of left ventricular ejection fraction (LVEF)). Our hypothesis was that ID would be prevalent in patients with severe AS, even in patients with normal haemoglobin. We also presumed that ID would be associated with increased morbidity and mortality in patients undergoing aortic valve replacement (AVR) as well as in patients not selected for surgery. We assessed a wide range of biomarkers of iron status beyond those that have been used for the definition of ID in patients with HF, e.g. hepcidin and soluble transferrin receptor that may be more reliable markers of ID in chronic disorders characterised by subclinical inflammation.

\section{METHODS}

\section{Study design and patient population}

This study is part of a prospective cohort study on survival, morbidity, functional status and QoL in consecutive patients with severe AS referred for evaluation for AVR at Oslo University Hospital Rikshospitalet in Norway between May 2010 and March 2013.22 23 Inclusion criteria were age $>18$ years, severe AS and the ability to read and write in Norwegian. Patients were excluded if they did not have severe AS, were unwilling to participate or had previously undergone AVR. Severe AS was defined in accordance with current guidelines. ${ }^{24}$ In cases of a low-flow, low-gradient state, with either preserved or reduced LVEF, patients were further evaluated by low-dose dobutamine stress and/or transoesophageal echocardiography. Patients were included before the decision as to whether to refer the patient to AVR or continued medical treatment was made and at follow-up after one year. By March 2017, mortality data were obtained from the national Norwegian Cause of Death Registry. Adverse clinical events and hospitalisation were assessed by reviewing medical records from Oslo University Hospital, where AVR was performed, and local hospitals in the year after inclusion. The endpoints for this study were all-cause mortality during short-term (one-year) and long-term (median 4.7 years, IQR: 3.8-5.5) follow-up and major adverse cardiovascular events (MACE) within the first year after surgery or inclusion. The composite endpoint MACE comprised transient ischaemic attack (TIA), stroke, myocardial infarction (MI) or all-cause death. All patients gave written informed consent before study participation.

Healthy subjects were either acquaintances of the staff, or they were recruited through announcements at the local blood bank (Blodbanken i Oslo) and at a local centre for the elderly. The inclusion criteria for healthy subjects were age $>18$ years, no signs or symptoms of cardiovascular disease, absence of any acute or chronic illness and no medications.

\section{Clinical data}

All patients underwent clinical and physical examinations at baseline and at follow-up after one year, including resting blood pressure evaluation, 12-lead electrocardiogram (ECG), peripheral blood sampling, six-minute walk test and transthoracic echocardiography.

\section{Blood sampling and biochemistry}

Peripheral venous blood was collected in tubes without any additives, allowed to coagulate for 60 minutes and centrifuged at $1500 \mathrm{~g}$ for 12 minutes. The serum was aliquoted into multiple cryo tubes and stored at $-80^{\circ} \mathrm{C}$ until analysis. The serum concentrations of ferritin, iron, transferrin, total iron-binding capacity, transferrin saturation, soluble transferrin receptor and hepcidin were assessed as markers of iron status. Serum hepcidin and soluble transferrin receptor were measured using commercially available antibodies in an enzyme immune assay (R\&D systems, Stillwater, Minnesota, USA), and the rest were measured by a Roche Modular P and E system.

The definition of anaemia was based on gender-specific cut-off values, as recommended by the World Health Organization (WHO) (haemoglobin $<13.0 \mathrm{~g} / \mathrm{dL}$ for men, $<12.0 \mathrm{~g} / \mathrm{dL}$ for women). ${ }^{25}$ Haematological variables were assessed in fresh EDTA blood by the Sysmex automatic analyser.

\section{Transthoracic echocardiography}

Echocardiography was performed using Vivid 7 or E9 ultrasound scanners (GE Vingmed Ultrasound, Horten, Norway). The maximal aortic jet velocity was measured using continuous-wave Doppler ultrasound through multiple acoustic windows. The maximal instantaneous and mean pressure gradients across the aortic valve were measured using the time velocity integral, and the aortic valve area was calculated using the continuity equation. The modified biplane Simpson was used to calculate LVEF. $^{26}$ 


\section{Diagnosis and classification of ID}

When assessing the association between ID and mortality and MACE, we applied a consensus definition of ID used in patients with $\mathrm{HF}^{16}$ Tentatively, we also used the 5th percentile and lower quartile for hepcidin and the 95th percentile and upper quartile for soluble transferrin receptor, respectively, as markers of ID. Reference values for soluble transferrin receptor and hepcidin, from which the values for the percentiles were derived, were measured in 50 healthy subjects. As there is no validated definition of ID in patients with severe AS, we also examined the association between iron, ferritin, soluble transferrin receptor, hepcidin and transferrin saturation as continuously z-score log-transformed variables and one-year and long-term mortality.

\section{Statistical analysis}

Data analyses were performed using IBM SPSS V.25. Baseline data are expressed as means with standard deviation (SD), as medians with interquartile range (IQR) or as numbers and percentages depending on distribution form. Between-group differences were tested using the Student's $t$-test, Mann-Whitney U test or Pearson $\chi^{2}$ test when appropriate. We assessed determinants of ID in a multivariable logistic regression model. The variables presented in table 1 were evaluated, and those with a significant univariable association with ID $(p<0.20)$ were entered in a stepwise backward multivariable model based on the strength of their univariable association. Logarithmic transformation was performed to achieve normal distributions for skewed variables, such as N-terminal pro-B-type natriuretic peptide (NT-proBNP) and troponin $\mathrm{T}$. When performing survival analysis, we divided the patients in groups of operated and conservatively treated patients. All analyses were performed according to the initial treatment strategy. Kaplan-Meier curves were constructed to demonstrate the effect of baseline markers of ID on cumulative survival. Differences in event-free survival rates were tested using the Cox-Mantel log-rank test. We performed Cox proportional hazard regression analyses to examine the associations between these variables and the risk of mortality during short-term and long-term follow-up, and the composite endpoint MACE within one year after inclusion. Baseline variables included in multivariable models were selected based on existing literature ${ }^{27} 28$ and specified prior to testing. For mortality analysis, we adjusted for age, gender, New York Heart Association (NYHA) class III and IV, LVEF, CAD, diabetes mellitus and atrial fibrillation. For analysis on MACE, we adjusted for age, NYHA class III and IV, LVEF, CAD and diabetes mellitus. All tests were two-sided, and p-values $<0.05$ were considered statistically significant.

\section{RESULTS}

\section{Clinical characteristics}

Of 573 consecutive, eligible patients referred for evaluation of severe AS, 68 declined participation, 20 had moderate AS and 5 patients received another diagnosis. In all, 480 patients were enrolled in the cohort study, of whom 16 patients had an insufficient amount of serum stored in the biobank for analyses of iron parameters (figure 1). Among the 464 patients who were eligible for this substudy, 91 patients $(20 \%)$ received conservative treatment due to either a lack of symptoms $(n=34)$, refusal to undergo surgery $(n=20)$ or either a high riskbenefit ratio or another disease that significantly reduces life expectancy $(n=37)$. The remaining 373 patients $(80 \%)$ underwent either surgical aortic valve implantation $(n=337)$ or transcatheter aortic valve implantation (TAVI) $(\mathrm{n}=36)$ at Oslo University Hospital, Rikshospitalet. The characteristics of patients who underwent AVR and those of conservatively treated patients have been reported previously showing that patients who underwent surgery were younger, had a higher NYHA and Canadian Cardiovascular Society class, had fewer comorbidities and used less medication. ${ }^{23}$

Baseline characteristics for all 464 patients and the 50 healthy volunteers are shown in table 1 . Patients with severe AS had significantly higher serum levels of hepcidin, lower haemoglobin and higher NT-proBNP than the controls (all $\mathrm{p}<0.05)$. Based on the definition applied in patients with HF, ID was present in 246 patients (53\%). 94 patients $(20 \%)$ had anaemia. Patients with anaemia were more often iron deficient than non-anaemic patients ( $63 \%$ vs $51 \%$ respectively, $\mathrm{p}=0.03$ ). Patients with ID had an overall worse clinical profile with older age $(76 \pm 10$ vs $74 \pm 11$ years, $\mathrm{p}=0.011$ ), higher median EuroSCORE II (3, IQR: 2-5 vs 2, IQR: 1-4, $\mathrm{p}=0.003)$, shorter distance on the six-minute walk test $(413 \pm 133$ vs $467 \pm 135 \mathrm{~m}, \mathrm{p}=0.002)$, lower Physical Component Summary score on the Short Form-36 QoL questionnaire ( $37 \pm 11$ vs $40 \pm 10, \mathrm{p}=0.008$ ), smaller aortic valve area $\left(0.63 \pm 0.17\right.$ vs $0.72 \pm 0.23 \mathrm{~cm}^{2}$, $\mathrm{p}<0.001$ ) and higher NT-proBNP (155, IQR: 44-258 vs 72, IQR: 29-241 pmol/L, p=0.01) than patients without ID. Patients with ID were also more frequently women (54\% vs $32 \%, \mathrm{p}<0.001$ ) and used more proton-pump inhibitors (PPIs)/antacids ( $18 \%$ vs $9 \%, \mathrm{p}=0.003)$ and less ACE inhibitor (ACEi)) / angiotensin receptor blocker (ARB) $(45 \%$ vs $35 \%, \mathrm{p}=0.028)$.

Stepwise backward logistic regression identified female sex (OR: 1.70 (95\% CI 1.11 to 2.61)), use of PPI/antacids (OR: 2.32 (95\% CI 1.26 to 4.30$)$ ), smaller aortic valve area (OR: 0.84 (95\% CI 0.75 to 0.93$)$ per $0.1 \mathrm{~cm}^{2}$ ), lower haemoglobin (OR: 0.78 (95\% CI 0.68 to 0.90$)$ ) and no use of ACEi/ARB (OR: 0.59 (95\% CI 0.39 to 0.90$)$ ) as independent predictors of ID (all p<0.05) (online supplementary table 1 ).

Of the 94 patients with anaemia, 59 (63\%) had ID. In the remaining 35 patients, we found a possible explanation for the anaemia in 13 patients (rheumatic disease, active cancer, kidney disease (estimated glomerular filtration rate $<60 \mathrm{~mL} / \mathrm{min} / 1.73 \mathrm{~m}^{2}$ ) and history of gastrointestinal bleeding). 
Table 1 Baseline characteristics of the entire cohort, according to the presence of ID, and of the healthy volunteers

\begin{tabular}{|c|c|c|c|c|c|}
\hline \multirow[b]{2}{*}{ Variables, units } & \multicolumn{4}{|c|}{ Patients with severe aortic stenosis } & \multirow{2}{*}{$\begin{array}{l}\text { Healthy } \\
\text { volunteers } \\
(n=50)\end{array}$} \\
\hline & $\begin{array}{l}\text { All patients } \\
(n=464)\end{array}$ & $\begin{array}{l}\text { Severe AS and } \\
I D^{*}(n=246)\end{array}$ & $\begin{array}{l}\text { Severe AS and } \\
\text { no ID* }(n=218)\end{array}$ & $\begin{array}{l}\text { P value for } \\
\text { difference }\end{array}$ & \\
\hline \multicolumn{6}{|l|}{ Demography } \\
\hline Age, years & $75(11)$ & $76(10)$ & 74 (11) & 0.011 & $67(8) \ddagger$ \\
\hline Female sex, n (\%) & $202(44)$ & $133(54)$ & $69(32)$ & $<0.001$ & $18(36)$ \\
\hline Body mass index, $\mathrm{kg} / \mathrm{m}^{2}$ & $26(5)$ & $26(5)$ & $26(4)$ & 0.889 & $25(3)$ \\
\hline Current smoker, n (\%) & $59(13)$ & $33(13)$ & $26(12)$ & 0.631 & \\
\hline \multicolumn{6}{|l|}{ Medical history, n (\%) } \\
\hline Hypertension & $208(45)$ & $108(44)$ & $100(46)$ & 0.670 & \\
\hline Heart failure & $34(7)$ & $21(9)$ & $13(6)$ & 0.288 & \\
\hline Atrial fibrillation/flutter, all types & $106(23)$ & $58(24)$ & $48(22)$ & 0.690 & \\
\hline Diabetes mellitus type I and II & $57(12)$ & $37(15)$ & $20(9)$ & 0.055 & \\
\hline Pulmonary disease & $87(19)$ & $48(20)$ & $39(18)$ & 0.672 & \\
\hline Kidney disease, eGFR $<60 \mathrm{~mL} / \mathrm{min} / 1.73 \mathrm{~m}^{2}$ & $31(7)$ & $17(7)$ & $14(6)$ & 0.833 & \\
\hline Coronary artery disease & $150(32)$ & $86(35)$ & $64(29)$ & 0.198 & \\
\hline Malignancy (previous/active) & $58(13)$ & $37(15)$ & $21(10)$ & 0.079 & \\
\hline \multicolumn{6}{|l|}{ Medication, n (\%) } \\
\hline Beta blocker & $223(48)$ & $122(50)$ & $101(46)$ & 0.483 & \\
\hline ACEi/ARB & $186(40)$ & $87(35)$ & $99(45)$ & 0.028 & \\
\hline Calcium antagonist & $92(20)$ & $47(19)$ & $45(21)$ & 0.679 & \\
\hline Statin & $249(54)$ & $127(52)$ & $122(56)$ & 0.350 & \\
\hline Diuretic & $176(38)$ & $90(37)$ & $86(39)$ & 0.526 & \\
\hline Anticoagulants/antiplatelets & $336(72)$ & $174(71)$ & $162(74)$ & 0.389 & \\
\hline Antacids/PPI & $46(14)$ & $45(18)$ & $19(9)$ & 0.003 & \\
\hline \multicolumn{6}{|l|}{ Clinical findings } \\
\hline Systolic blood pressure, mm Hg & $143(23)$ & $144(23)$ & $142(23)$ & 0.327 & \\
\hline Diastolic blood pressure, $\mathrm{mm} \mathrm{Hg}$ & $77(12)$ & $77(13)$ & $77(11)$ & 0.325 & \\
\hline Heart rate, beats/min & $73(13)$ & $73(14)$ & $72(12)$ & 0.979 & \\
\hline \multicolumn{6}{|l|}{ Surgical risk score } \\
\hline EuroSCORE II & $3(1-5)$ & $3(2-5)$ & $2(1-4)$ & 0.003 & \\
\hline Six-minute walk test, meters & $436(136)$ & $413(133)$ & 467 (135) & 0.002 & \\
\hline \multicolumn{6}{|l|}{ Health-related quality of life } \\
\hline Summary PCS (sf36-v2) & $38(11)$ & $37(11)$ & $40(10)$ & 0.008 & \\
\hline Summary MCS (sf36-v2) & $50(12)$ & $49(12)$ & $50(12)$ & 0.904 & \\
\hline EQ-5D UK index score, median (IQR) & $0.70(0.63-0.77)$ & $0.71(0.66-0.77)$ & $0.70(0.60-0.77)$ & 0.160 & \\
\hline EQ-VAS score & $58(22)$ & $57(22)$ & $60(21)$ & 0.061 & \\
\hline \multicolumn{6}{|l|}{ Cognitive ability } \\
\hline MMSE, median (IQR) & $29(28-30)$ & $29(27-30)$ & $29(28-29)$ & 0.926 & \\
\hline Charlson comorbidity index, $\mathrm{n}(\%)$ & & & & 0.131 & \\
\hline 0 & $177(38)$ & $85(35)$ & $92(42)$ & & \\
\hline $1-2$ & $226(49)$ & $123(50)$ & $103(47)$ & & \\
\hline$\geq 3$ & $61(13)$ & $38(15)$ & $23(11)$ & & \\
\hline NYHA classification, $n(\%)$ & & & & 0.636 & \\
\hline Class I & $51(11)$ & $24(10)$ & $27(12)$ & & \\
\hline Class II & $200(43)$ & $106(43)$ & $94(43)$ & & \\
\hline
\end{tabular}


Table 1 Continued

\begin{tabular}{|c|c|c|c|c|c|}
\hline \multirow[b]{2}{*}{ Variables, units } & \multicolumn{4}{|c|}{ Patients with severe aortic stenosis } & \multirow{2}{*}{$\begin{array}{l}\text { Healthy } \\
\text { volunteers } \\
(n=50)\end{array}$} \\
\hline & $\begin{array}{l}\text { All patients } \\
(n=464)\end{array}$ & $\begin{array}{l}\text { Severe AS and } \\
I D^{\star}(n=246)\end{array}$ & $\begin{array}{l}\text { Severe AS and } \\
\text { no ID* }(n=218)\end{array}$ & $\begin{array}{l}\text { P value for } \\
\text { difference }\end{array}$ & \\
\hline Class III/IV & $213(46)$ & $116(47)$ & $97(45)$ & & \\
\hline CCS, angina grade, $n(\%)$ & & & & 0.166 & \\
\hline Score 0 & $263(57)$ & $140(57)$ & $123(57)$ & & \\
\hline Score 1-2 & $172(37)$ & $86(35)$ & $86(39)$ & & \\
\hline Score 3-4 & $29(6)$ & $20(8)$ & $9(4)$ & & \\
\hline \multicolumn{6}{|l|}{ Echocardiographic measures } \\
\hline BSA & $1.88(0.22)$ & $1.85(0.22)$ & $1.92(0.22)$ & $<0.001$ & \\
\hline LVEF, \% & $56(9)$ & $56(9)$ & $57(9)$ & 0.301 & \\
\hline Aortic peak velocity, m/s & $4.7(07)$ & $4.7(0.7)$ & $4.7(0.7)$ & 0.766 & \\
\hline Aortic mean gradient, $\mathrm{mm} \mathrm{Hg}$ & $55(18)$ & $55(18)$ & $55(18)$ & 0.876 & \\
\hline Aortic valve area, $\mathrm{cm}^{2}$ & $0.67(0.20)$ & $0.63(0.17)$ & $0.72(0.23)$ & $<0.001$ & \\
\hline \multicolumn{6}{|l|}{ Biochemistry } \\
\hline Haemoglobin, g/dL & $13.6(1.6)$ & $13.2(1.6)$ & $14.0(1.5)$ & $<0.001$ & $14.3(0.9) \ddagger$ \\
\hline NT-pro-BNP, pmol/L, median (IQR) & $102(36-252)$ & $115(44-258)$ & $72(29-241)$ & 0.011 & $8(8-28) \ddagger$ \\
\hline hs-TnT, ng/mL, median (IQR) & $14(10-25)$ & $14(10-27)$ & $12(10-25)$ & 0.188 & \\
\hline Creatinine, $\mu \mathrm{mol} / \mathrm{L}$, median (IQR) & $81(68-99)$ & $82(67-101)$ & $81(69-95)$ & 0.600 & \\
\hline CRP, mg/L, median (IQR) & $2.1(0.8-5.4)$ & $2.4(0.8-6.3)$ & $1.8(0.7-4.9)$ & 0.093 & \\
\hline Ferritin, $\mu \mathrm{g} / \mathrm{L}$, median (IQR) & $117(66-197)$ & $69(41-93)$ & $187(136-280)$ & $<0.001$ & \\
\hline Transferrin, g/L & $2.6(0.7)$ & $2.7(0.9)$ & $2.4(0.4)$ & $<0.001$ & \\
\hline Iron, $\mu \mathrm{mol} / \mathrm{L}$, median (IQR) & $14(11-19)$ & $11(9-15)$ & $17(14-20)$ & $<0.001$ & \\
\hline Total iron binding capacity (TIBC) & $64(11)$ & $67(11)$ & $50(9)$ & $<0.001$ & \\
\hline Transferrin saturation (TSAT) (\%) median (IQR) & $23(17-30)$ & $18(13-23)$ & $28(24-34)$ & $<0.001$ & \\
\hline Hepcidin, ng/mL, median (IQR) & $36.2(16.4-59.3)$ & $22.3(11.2-44.9)$ & $51.4(29.0-71.4)$ & $<0.001$ & $16.0(6.4-41.5) \ddagger$ \\
\hline Transferrin receptor, ng/mL, median (IQR) & $777(620-1005$ & $883(689-1158$ & $703(573-848)$ & $<0.001$ & $744(619-950)$ \\
\hline Presence of anaemia, $\mathrm{n}(\%)$ & $94(20)$ & $59(24)$ & $35(16)$ & 0.029 & \\
\hline Presence of ferritin <30 $\mu \mathrm{g} / \mathrm{L}, \mathrm{n}(\%)$ & $31(7)$ & $31(13)$ & $0(0)$ & $<0.001$ & \\
\hline Presence of ID, n (\%) & $246(53)$ & $246(100)$ & $0(0)$ & $<0.001$ & \\
\hline Absolute (ferritin <100 $\mu \mathrm{g} / \mathrm{L}), \mathrm{n}(\%)$ & $195(42)$ & $195(79)$ & $0(0)$ & $<0.001$ & \\
\hline $\begin{array}{l}\text { Functional (ferritin 100-300 } \mu \mathrm{g} / \mathrm{L} \text { and low TSAT } \\
(<20 \%)), \mathrm{n}(\%)\end{array}$ & $51(11)$ & $51(21)$ & $0(0)$ & $<0.001$ & \\
\hline
\end{tabular}

Selected characteristics of the study sample $(n=464)$. The numbers are mean (SD), frequency (\%) or medians (IQR). Complete data of ferritin, transferrin, iron, total iron binding capacity (TIBC), transferrin saturation (TSAT) and hepcidin. Other numbers may vary due to missing values. P-values for comparison of results of patients with ID and those without ID.

*ID was defined as ferritin level $<100 \mu \mathrm{g} / \mathrm{L}$ or ferritin $100-299 \mu \mathrm{g} / \mathrm{L}$ with a transferrin saturation $<20 \%$.

†Anaemia was defined as haemoglobin level $<12 \mathrm{~g} / \mathrm{dL}$ in women and $<13 \mathrm{~g} / \mathrm{dL}$ in men. $\ddagger \mathrm{P}<0.001$, patients with severe AS (all patients) compared with healthy subjects.

ACEi, ACE inhibitor; ARB, angiotensin receptor blocker; AS, Aortic stenosis; BSA, body surface area; CCS, Canadian Cardiovascular Society; CRP, C-reactive protein; EQ-5D UK, EuroQol 5-dimension United Kingdom; EQ-VAS, EuroQol Visual Analogue Scale; ID, Iron deficiency; LVEF, left ventricular ejection fraction; MCS, Mental Component Summary; MMSE, Mini-Mental State Examination; NT-proBNP, N-terminal pro-B-type natriuretic peptide; NYHA, New York Heart Association; PCS, Physical Component Summary; PPI, proton-pump inhibitor; eGFR, estimated glomerular filtration rate; hsTNT, high-sensitive troponin T; sf36-v2, Short Form Health Survey version 2.0.

\section{Hepcidin and soluble transferrin receptor}

45 patients $(10 \%)$ had a soluble transferrin receptor concentration above the 95 th percentile $(\geq 1308 \mathrm{ng} / \mathrm{mL})$ and 138 patients $(30 \%)$ had a soluble transferrin receptor level in the upper quartile $(\geq 950 \mathrm{ng} / \mathrm{mL}) .8$ patients $(2 \%)$ had a hepcidin level below the 5 th percentile $(\leq 1.0$ $\mathrm{ng} / \mathrm{mL}$ ) and 34 patients $(7 \%)$ had a hepcidin concentration in the lower quartile $(<6.4 \mathrm{ng} / \mathrm{mL})$. Patients with ID had a significantly higher concentration of soluble transferrin receptor $(883 \mathrm{ng} / \mathrm{mL}$, IQR:689-1160 vs 703 $\mathrm{ng} / \mathrm{mL}$, IQR:573-848, $\mathrm{p}<0.001)$ and a significantly lower concentration of hepcidin (22 ng/mL, IQR:11-45 vs 51 


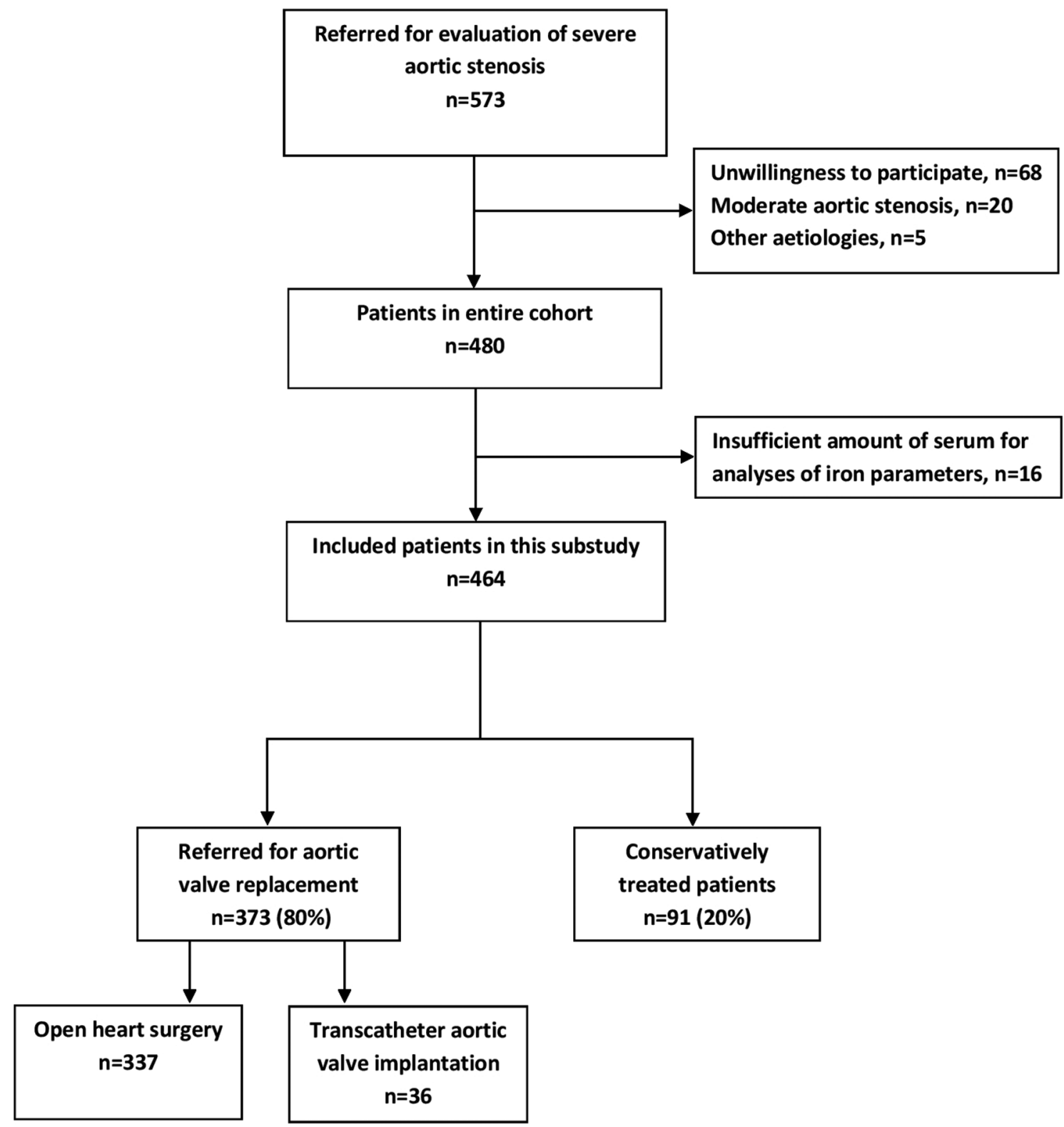

Figure 1 Study flow.

$\mathrm{ng} / \mathrm{mL}, \mathrm{IQR}: 29-71), \mathrm{p}<0.001)$ than patients without ID (figure 2).

\section{Survival}

The mean duration of follow-up in all 464 patients was $4.3( \pm 1.7)$ years (median 4.7 years, IQR: $3.8-5.5)$. During this period 129 patients $(29 \%)$ died, of whom 57 were in the conservatively treated group $(63 \%$ of patients in this group) and 72 in the group who had AVR (19\% of patients in this group). 70 deaths (54\% of all deaths) were caused by cardiovascular disease. In the remaining 59 patients, the causes of death were as follows: pulmonary disease including pneumonia: 19 , malignancy: 14 , stroke: 9, infection other than pneumonia: 7 , hip fracture: 5, ruptured abdominal aortic aneurysm: 2, kidney disease: 2, Alzheimer's disease: 1.38 deaths (29\% of all deaths) occurred within the first year after inclusion.
Overall ID (as defined in patients with HF) was not associated with mortality (HR: 1.14 (95\% CI 0.81-1.62), $\mathrm{p}=0.46$ for long-term mortality and HR: 1.24 (95\% CI $0.65-2.35), p=0.52$ for one-year mortality). There was no significant difference in long-term mortality between patients with and without ID in either the operated or the conservatively treated group $(\log$-rank $\mathrm{p}=0.25$ and $\mathrm{p}=0.83$ for the operated (figure $3 \mathrm{~A}$ ) and the conservatively treated patients, respectively). Nor was there any significant difference in one-year mortality between patients with and without ID in either group (log-rank $p=0.23$ and $p=0.86$ for the operated and the conservatively (figure 3C) treated patients, respectively). Online supplementary table 2 shows the number of patients and events in each group. ID was not an independent predictor of long-term mortality $(\mathrm{p}=0.88$ and $\mathrm{p}=0.84$ in 

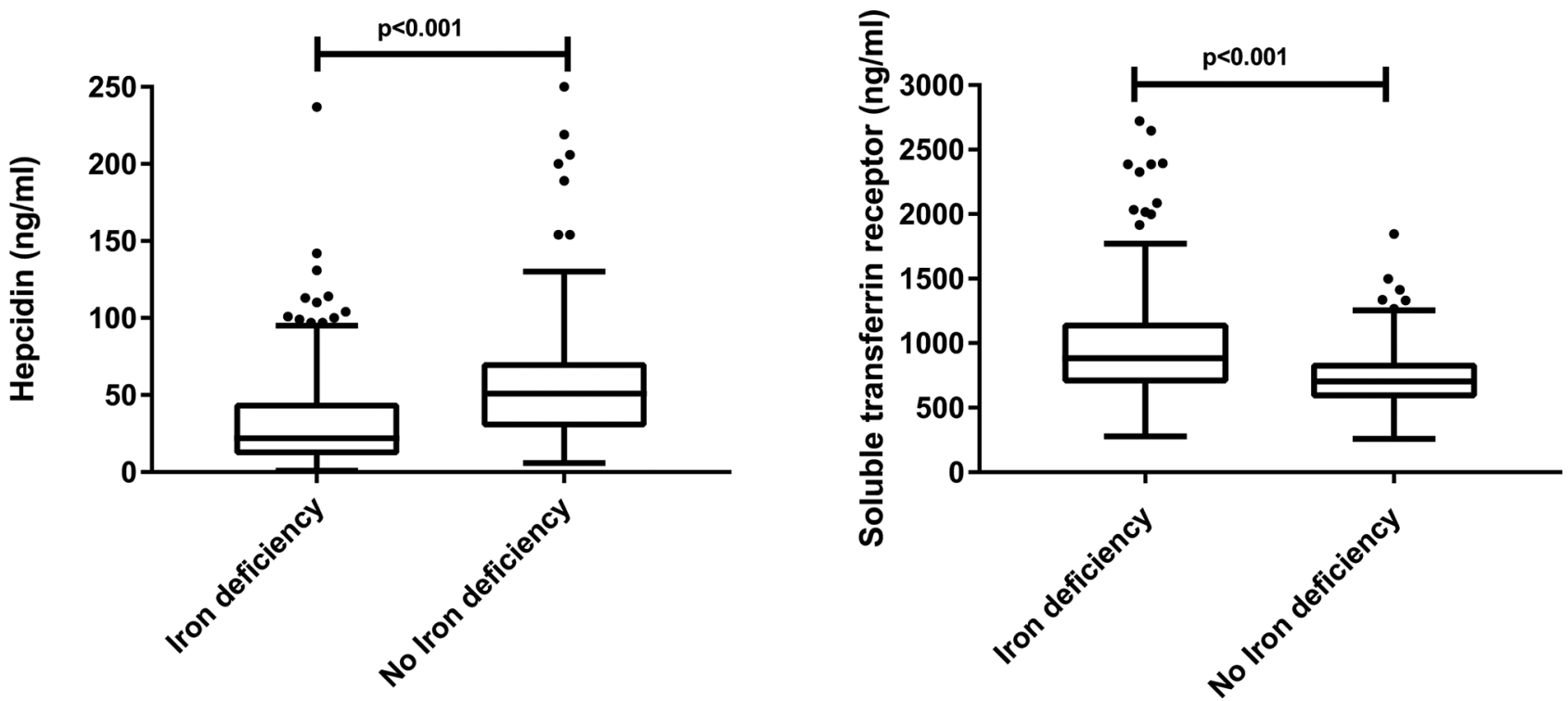

Figure 2 Comparison of hepcidin (A) and soluble transferrin receptor (B) levels between patients with and without iron deficiency.

the operated and conservatively treated patients, respectively) or one-year mortality $(\mathrm{p}=0.29$ and $\mathrm{p}=0.31$ in the operated and conservatively treated patients, respectively) after adjustment for predetermined clinical and echocardiographic predictors. The severity of the AS, as estimated by the aortic valve area, was not a significant confounder on top of the prespecified variables. In the subset of patients with an LVEF $<50(17 \%)$, there
A

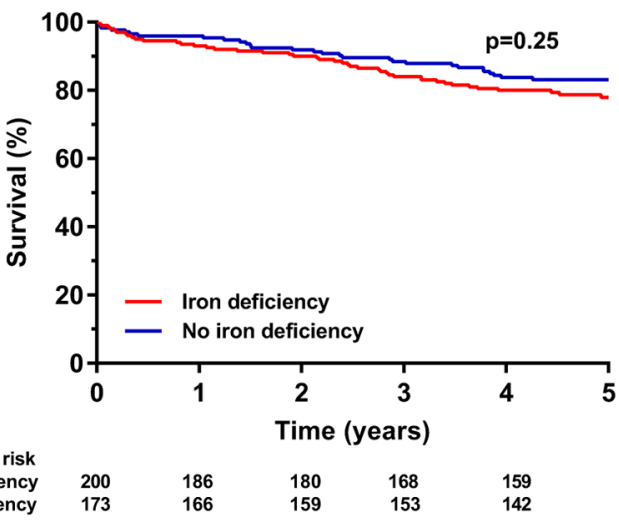

C

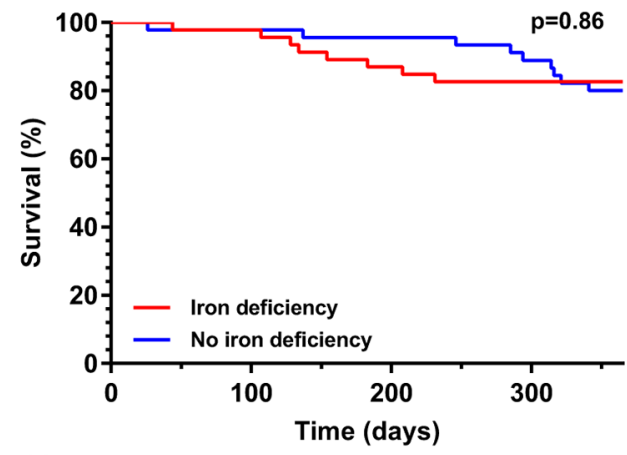

B

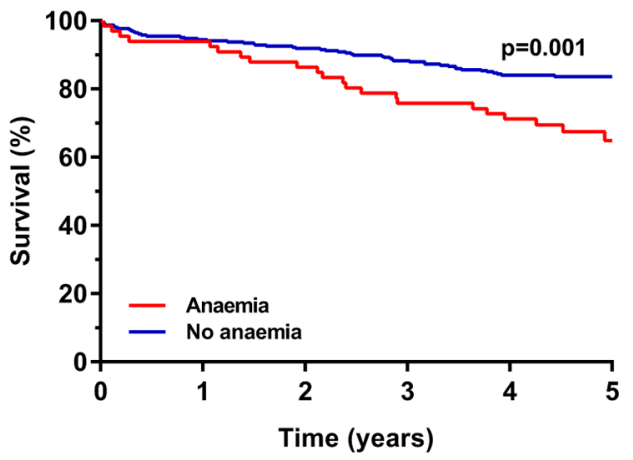

$\begin{array}{rccccc}\begin{array}{c}\text { Number at risk } \\ \text { Anaemia }\end{array} & 66 & 62 & 57 & 50 & 41 \\ \text { No anaemia } & 307 & 290 & 282 & 271 & 206\end{array}$

D

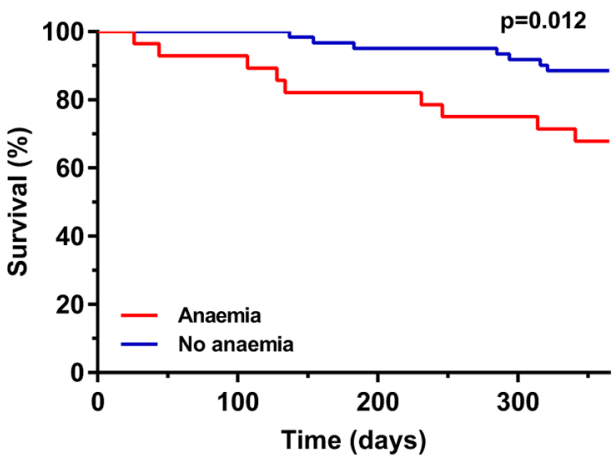

Number at risk

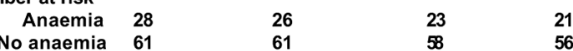


was no difference in long-term survival in either groups (log-rank $\mathrm{p}=0.91$ and $\mathrm{p}=0.85$ for the operated and the conservatively treated patients, respectively).

A low serum hepcidin level (below the lower quartile in the healthy control group) and a high-soluble transferrin receptor concentration (above the upper quartile in the healthy control group) were not associated with long-term mortality overall $(\log$-rank $\mathrm{p}=0.52$ and $\mathrm{p}=0.14$ for hepcidin and transferrin receptor, respectively), or in the conservatively treated patients $(\log$-rank $\mathrm{p}=0.65$ and $\mathrm{p}=0.74$ for hepcidin and soluble transferrin receptor, respectively) or in the patients who underwent surgery (log-rank $\mathrm{p}=0.51$ and $\mathrm{p}=0.23$ for hepcidin and soluble transferrin receptor, respectively). Nor were they associated with one-year mortality in either group. A serum hepcidin level below the 5 th percentile and soluble transferrin receptor concentration above the 95th percentile in the healthy control group was not associated with either long-term or short-term mortality in either group.

When assessed as continuous variables, serum iron and transferrin saturation were associated with long-term mortality in patients who underwent surgery (HR: 0.77 (95\% CI 0.62-0.97), $\mathrm{p}=0.024$ and HR: 0.78 (95\% CI 0.62$0.98), \mathrm{p}=0.032$, respectively) in univariable Cox regression analyses. This association did not remain significant when adjusting for the predetermined confounding factors. Soluble transferrin receptor, hepcidin and ferritin were not associated with long-term mortality when assessed as continuous variables in univariable Cox regression analyses. None of the five above mentioned markers of ID were associated with one-year mortality.

Anaemia was associated with long-term mortality in patients who underwent AVR (log-rank $\mathrm{p}=0.001$ ) (figure 3B) but not in the conservatively treated group (log-rank $\mathrm{p}=0.14)$. Conversely, anaemia was associated with one-year mortality in the conservatively treated group ( $\log$-rank $\mathrm{p}=0.012$ ) (figure $3 \mathrm{D}$ ), but not in the operated group $(\log -$ rank $\mathrm{p}=0.85)$. In multivariable analyses, anaemia remained an independent predictor of one-year mortality in the conservatively treated patients (HR: 4.58 (95\% CI 1.30-16.1), $\mathrm{p}=0.018$ ) (online supplementary table 3$)$.

\section{Major adverse cardiovascular events}

The composite endpoint MACE occurred in 73 patients within the first year after inclusion (stroke/TIA: 33, all-cause death: 25, MI: 15). 21 patients (23\%) in the conservatively treated group and 52 patients (14\%) in the group undergoing AVR experienced MACE. There were no significant differences in the event rates between patients with and without ID or anaemia in either treatment group. ID (online supplementary table 4), anaemia, low hepcidin or high-soluble transferrin receptor were not independent predictors of MACE in multiple analyses adjusted for predetermined predictors in either group.

The number of patients in the operated group hospitalised for $\mathrm{HF}$ at least once during the one-year observation period was $18(9 \%)$ in the group with ID versus 11
(6\%) for the group without ID $(\mathrm{p}=0.34)$. In the conservatively treated patients, the number of hospitalisations due to HF was 12 (26\%) in the group with ID and 9 $(20 \%)$ in the group without ID $(\mathrm{p}=0.49)$. There was no significant difference in the number of hospitalisation due to HF between patients with and without anaemia ( $p=0.34$ and $p=0.20$ in the surgically and conservatively treated patients, respectively). In the group undergoing aortic valve intervention, 11 patients $(6 \%)$ with ID and 10 patients $(6 \%)$ without ID experienced life-threatening bleeding events in the perioperative phase $(\mathrm{p}=0.91)$ There was no significant difference in the proportion of life-threatening bleeding events between patients with and without anaemia $(\mathrm{p}=0.67)$.

\section{DISCUSSION}

This prospective study shows that both ID, as defined in patients with HF, and anaemia are common comorbidities in patients with severe AS, with a prevalence of $53 \%$ and $20 \%$, respectively. The prevalence of anaemia in our patients was relatively low compared with what has been observed in recent studies on patients with AS mainly referred for TAVI, where the prevalence of anaemia has been reported to be between $22 \%$ and $57 \% .^{18-21}$ This is most likely explained by differences in patient characteristics. The current cohort included consecutive patients with severe AS who were referred to our tertiary centre for evaluation for possible AVR between 2010 and 2013. Some of the elderly, frail patients referred to TAVI today may not have been referred for surgery at the time. However, a substantial proportion of our patients were deemed too frail for aortic valve intervention (8\%), treated with TAVI $(8 \%)$ or categorised as asymptomatic $(7 \%)$. We are confident that our results apply to contemporary patients who are evaluated for AVR due to severe AS. ID was present in 91 of $115(79 \%)$ anaemic patients with AS referred for TAVI. ${ }^{19}$ The prevalence of ID regardless of anaemia in patients with AS has not been addressed before. Assuming that the definition of ID in HF patients is applicable in patients with AS, the prevalence of ID is high.

The main objective of our study was to assess the prognostic implications of ID and anaemia in patients with severe AS. Using the definition commonly applied in patients with HF, we found that patients with ID in general were more comorbid than patients without ID, but there were no significant associations between ID and mortality or MACE. Since the usefulness of the operational definition of ID applied in patients with HF is unknown in patients with other cardiovascular diseases, we investigated whether soluble transferrin receptor or hepcidin could provide additional prognostic value. ${ }^{29} 30$ These markers are not used as often due to their relatively short history and higher costs but have been proposed to be better markers for the determination of ID in patients with comorbidities and inflammation. ${ }^{31}$ In our study, however, neither soluble transferrin receptor nor 
hepcidin was associated with outcome. We also assessed soluble transferrin receptor, serum iron, ferritin, transferrin saturation and hepcidin as continuous variables and found only a univariable association between serum iron and transferrin saturation with long-term mortality in the patients who underwent surgery.

In this study, we have applied many definitions of ID, none of which predicted outcomes. We therefore feel confident that ID (by any definition) does not portend outcomes in patients with severe AS. Anaemia was associated with long-term mortality in patients who underwent AVR and with one-year mortality in conservatively treated patients. The latter association remained significant after adjustment for conventional risk factors.

The majority of our patients received AVR. The detrimental effect of ID and anaemia may be offset by blood transfusions. After surgery, the original disease (AS) is removed, and ID and anaemia may autocorrect or no longer have the same negative effect on survival. Patients with AS often have Heyde syndrome, a triad of AS, acquired coagulopathy (von Willebrand syndrome 2A) and anaemia due to bleeding from intestinal angiodysplasia. ${ }^{32}$ Since the acquired coagulopathy tends to correct itself and the anaemia tends to abate after valve replacement, ${ }^{32} 33$ surgery may attenuate the effect of anaemia on mortality. This would explain the lack of association between presurgical anaemia and mortality in the patients who underwent surgery. We can only speculate as to why anaemia was associated with short-term, but not long-term, mortality in the conservatively treated patients. Given the limited number of patients in this group, this result must be interpreted with caution.

Baseline characteristics show an association between ID and functional capacity and QoL. Patients with ID also have higher NT-proBNP. In a study by DeLarochellière $e t$ $a l,{ }^{34}$ anaemia was an independent predictor of a poorer performance in the six-minute walk test and worse QoL in patients scheduled for TAVI. Gómez et $a t^{20}$ showed in their study that the resolution of anaemia by treating ID was associated with improvements in echocardiographic parameters and functional status. These results suggest that low haemoglobin and maybe also ID limits physical capacity, is associated with reduced QoL and may represent a therapeutic target in patients with severe AS.

Patients with ID had smaller aortic valve area. Patients with ID were more frequently females and had smaller body surface area explaining this difference.

\section{Study limitations}

This is a prospective, single-centre, observational study. Biomarkers reflecting iron metabolism were measured at baseline only, and we have no data on how these values evolve over time. The majority of the patients had AVR, and outcome in patients undergoing surgery is complex and multifactorial. Unfortunately, we did not register data regarding patient-prosthesis matching. The results in the conservatively treated patients must be interpreted with care due to the heterogeneity of this group and the limited number of patients. Unfortunately, we did not record MACE beyond the first year of follow-up. Unfortunately, we have no data on the use of oral iron supplements at inclusion. In our opinion, the main weakness of this study is the uncertainty regarding determining true ID in patients with AS. However, we did a comprehensive analysis of markers reflecting iron metabolism, and it is reasonable to believe that we have been able to reflect iron status as correctly as possible.

\section{CONCLUSIONS}

ID, as defined in patients with HF, and aneamia are common in patients with severe AS. ID was associated with worse clinical profile, but not with mortality or MACE in our patients with severe AS, most of whom received AVR. Anaemia was associated with one-year mortality in conservatively treated patients only. More studies are required to confirm these findings and to determine how to correctly diagnose ID in patients with AS. In our opinion, the definition of ID in chronic cardiovascular disorders could be improved by using additional markers that are less influenced by inflammation than ferritin.

\section{Author affiliations}

${ }^{1}$ Faculty of Medicine, University of Oslo, Oslo, Norway

${ }^{2}$ KG Jebsen Centre for Cardiac Research and Center for Heart Failure Research, Oslo University Hospital Ullevål, Oslo, Norway

${ }^{3}$ Department of Cardiology, Oslo University Hospital Rikshospitalet, Oslo, Norway ${ }^{4}$ Research Institute for Internal Medicine, Oslo University Hospital Rikshospitalet, Oslo, Norway

${ }^{5}$ K.G. Jebsen - Thrombosis Research and Expertise Center (TREC), The Faculty of Health Sciences, UT - The Arctic University of Norway, Tromsø, Norway

${ }^{6}$ Section of Clinical Immunology and Infectious Diseases, Oslo University Hospital, Rikshospitalet, Oslo, Norway

${ }^{7}$ Department of Medical Biochemistry, Oslo University Hospital Rikshospitalet, Oslo, Norway

Contributors LG, KB, LM, AA, AlH, PA and ABK conceived and designed the analysis. AA, AIH, LG, KIP, LM, TU, AEM and ABK collected the data. ABK and KB analysed the data. $A B K$ wrote the manuscript with support from $K B$. All authors revised the work critically, read and approved the final manuscript and agree to be accountable for all aspects of the work. ABK is responsible for the overall content as guarantor.

Funding The authors have not declared a specific grant for this research from any funding agency in the public, commercial or not-for-profit sectors.

Competing interests None declared.

Patient consent for publication Not required.

Provenance and peer review Not commissioned; externally peer reviewed.

Data sharing statement No additional data are available.

Open access This is an open access article distributed in accordance with the Creative Commons Attribution Non Commercial (CC BY-NC 4.0) license, which permits others to distribute, remix, adapt, build upon this work non-commercially, and license their derivative works on different terms, provided the original work is properly cited, appropriate credit is given, any changes made indicated, and the use is non-commercial. See: http://creativecommons.org/licenses/by-nc/4.0/

\section{REFERENCES}

1. World Health Organization, 2017. Micronutrient deficiencies Iron deficiency anaemia. Available from: http://www.who.int/nutrition/ topics/ida/en/ (Cited 4 Dec 2017).

2. Arora NP, Ghali JK. Iron deficiency anemia in heart failure. Heart Fail Rev 2013;18:485-501. 
3. Zimmermann MB, Hurrell RF. Nutritional iron deficiency. Lancet 2007;370:511-20.

4. Andrews NC. Disorders of iron metabolism. N Engl J Med 1999;341:1986-95.

5. Klip IT, Comin-Colet J, Voors AA, et al. Iron deficiency in chronic heart failure: an international pooled analysis. Am Heart $J$ 2013:165:575-82.

6. Jankowska EA, Rozentryt P, Witkowska A, et al. Iron deficiency: an ominous sign in patients with systolic chronic heart failure. Eur Heart J 2010;31:1872-80.

7. McDonagh T, Macdougall IC. Iron therapy for the treatment of iron deficiency in chronic heart failure: intravenous or oral? Eur J Heart Fail 2015;17:248-62

8. Okonko DO, Mandal AK, Missouris CG, et al. Disordered iron homeostasis in chronic heart failure: prevalence, predictors, and relation to anemia, exercise capacity, and survival. J Am Coll Cardiol 2011;58:1241-51.

9. Anker SD, Comin Colet J, Filippatos G, et al. Ferric carboxymaltose in patients with heart failure and iron deficiency. $N$ Engl J Med 2009:361:2436-48.

10. Ponikowski P, van Veldhuisen DJ, Comin-Colet J, et al. Beneficial effects of long-term intravenous iron therapy with ferric carboxymaltose in patients with symptomatic heart failure and iron deficiencydagger. Eur Heart J 2015;36:657-68.

11. Grammer TB, Kleber ME, Silbernagel G, et al. Hemoglobin, iron metabolism and angiographic coronary artery disease (The Ludwigshafen Risk and Cardiovascular Health Study). Atherosclerosis 2014;236:292-300.

12. Ponikowska B, Suchocki T, Paleczny B, et al. Iron status and survival in diabetic patients with coronary artery disease. Diabetes Care 2013;36:4147-56.

13. Ruiter G, Lankhorst S, Boonstra A, et al. Iron deficiency is common in idiopathic pulmonary arterial hypertension. Eur Respir $J$ 2011;37:1386-91.

14. Viethen T, Gerhardt F, Dumitrescu D, et al. Ferric carboxymaltose improves exercise capacity and quality of life in patients with pulmonary arterial hypertension and iron deficiency: a pilot study. Int J Cardiol 2014;175:233-9.

15. Piednoir P, Allou N, Driss F, et al. Preoperative iron deficiency increases transfusion requirements and fatigue in cardiac surgery patients: a prospective observational study. Eur J Anaesthesiol 2011;28:796-801.

16. von Haehling S, Jankowska EA, van Veldhuisen DJ, et al. Iron deficiency and cardiovascular disease. Nat Rev Cardiol 2015;12:659-69.

17. Grote Beverborg N, Klip IT, Meijers WC, et al. Definition of iron deficiency based on the gold standard of bone marrow iron staining in heart failure patients. Circ Heart Fail 2018;11:e004519.

18. Ng AC, Kong WK, Kamperidis V, et al. Anaemia in patients with aortic stenosis: influence on long-term prognosis. Eur J Heart Fail 2015;17:1042-9.
19. Rheude T, Pellegrini C, Michel J, et al. Prognostic impact of anemia and iron-deficiency anemia in a contemporary cohort of patients undergoing transcatheter aortic valve implantation. Int J Cardiol 2017;244:93-9.

20. Gómez M, Ble M, Cladellas M, et al. Effect of correction of anemia on echocardiographic and clinical parameters in patients with aortic stenosis involving a three-cuspid aortic valve and normal left ventricular ejection fraction. Am J Cardiol 2015;116:270-4.

21. Nuis RJ, Sinning JM, Rodés-Cabau J, et al. Prevalence, factors associated with, and prognostic effects of preoperative anemia on short- and long-term mortality in patients undergoing transcatheter aortic valve implantation. Circ Cardiovasc Interv 2013;6:625-34.

22. Auensen A, Hussain Al, Falk RS, et al. Associations of brainnatriuretic peptide, high-sensitive troponin $\mathrm{T}$, and high-sensitive C-reactive protein with outcomes in severe aortic stenosis. PLoS One 2017;12:e0179304.

23. Auensen A, Hussain Al, Bendz B, et al. Morbidity outcomes after surgical aortic valve replacement. Open Heart 2017;4:e000588.

24. Baumgartner H, Falk V, Bax JJ, et al. ESC/EACTS Guidelines for the management of valvular heart disease. Eur Heart $J$ 2017;2017;38:2739-91.

25. Nutritional anaemias. Report of a WHO scientific group. World Health Organ Tech Rep Ser 1968;405:5-37.

26. Baumgartner H, Hung J, Bermejo J, et al. Echocardiographic assessment of valve stenosis: EAE/ASE recommendations for clinical practice. J Am Soc Echocardiogr 2009;22:1-23.

27. Vahanian A, Andreotti F, Alfieri O, et al. Guidelines on the management of valvular heart disease (version 2012). Eur Heart $J$ 2012;33:2451-96.

28. Bouma BJ, van Den Brink RB, van Der Meulen JH, et al. To operate or not on elderly patients with aortic stenosis: the decision and its consequences. Heart 1999;82:143-8.

29. Jankowska EA, von Haehling S, Anker SD, et al. Iron deficiency and heart failure: diagnostic dilemmas and therapeutic perspectives. Eur Heart J 2013;34:816-29.

30. Goodnough LT, Nemeth E, Ganz T. Detection, evaluation, and management of iron-restricted erythropoiesis. Blood 2010;116:4754-61.

31. Jankowska EA, Kasztura M, Sokolski M, et al. Iron deficiency defined as depleted iron stores accompanied by unmet cellular iron requirements identifies patients at the highest risk of death after an episode of acute heart failure. Eur Heart J 2014;35:2468-76.

32. Massyn MW, Khan SA. Heyde syndrome: a common diagnosis in older patients with severe aortic stenosis. Age Ageing 2009;38:267-70.

33. Vincentelli A, Susen S, Le Tourneau T, et al. Acquired von Willebrand syndrome in aortic stenosis. N Engl J Med 2003;349:343-9.

34. DeLarochellière $\mathrm{H}$, Urena $\mathrm{M}$, Amat-Santos IJ, et al. Effect on outcomes and exercise performance of anemia in patients with aortic stenosis who underwent transcatheter aortic valve replacement. Am J Cardiol 2015;115:472-9. 